\title{
Tratamento de angina mesentérica em pacientes com arterite de Takayasu
}

Luana Thayse Barros de Lima ${ }^{1}$, Georges Basile Christopoulos², Virgínia Moreira Braga ${ }^{1}$, Maria Eliza M. Nemézio ${ }^{1}$, Ana Paula M.M. Souza ${ }^{1}$, Ana Clara C. Rêgo ${ }^{1}$

\begin{abstract}
RESUMO
A arterite de Takayasu (AT) é uma doença inflamatória crônica do tecido conectivo, idiopática, que acomete preferencialmente a aorta e seus ramos. A terapêutica utilizada baseia-se sobretudo no uso de corticosteroides e imunossupressores. É relatado o caso de uma paciente, 33 anos, com mal-estar, febre, mialgia, cefaleia intensa, pulsátil, holocraniana, resistente a analgésicos, hipertensão arterial sistêmica de difícil controle, claudicação no membro inferior direito e dor abdominal de forte intensidade, a qual piorava após a alimentação. A angiotomografia revelou aneurisma da aorta ascendente, estenose da artéria ilíaca comum direita, estenose das artérias renais e estenose da artéria mesentérica superior, fato que embasou o diagnóstico de angina mesentérica e a conduta intervencionista através da angioplastia transluminal percutânea múltipla com a colocação de stents.
\end{abstract}

Palavras-chave: arterite de Takayasu, angioplastia transluminal percutânea, dor abdominal.

[Rev Bras Reumatol 2011;51(2):188-195] CElsevier Editora Ltda.

\section{INTRODUÇÃO}

A arterite de Takayasu (AT) é uma doença inflamatória crônica do tecido conectivo, por vezes focal, idiopática, que acomete preferencialmente a aorta e seus ramos. Com distribuição universal, apresenta incidência maior em asiáticos e mulheres até a quarta década de vida. ${ }^{1}$ Os achados vasculares típicos incluem diminuição ou ausência de pulsos; diferença na aferição da pressão arterial em membros superiores; claudicação; hipertensão arterial sistêmica (HAS) de difícil controle; dor na área das artérias acometidas, além de sopros nas áreas de inflamação crônica ou estenose dos vasos de grande e médio calibres. Histopatologicamente caracteriza-se por uma inflamação granulomatosa que cursa com atrofia da camada média arterial e hipertrofia da camada íntima. Geralmente manifesta-se por lesões estenóticas de instalação abrupta. A ocorrência de aneurismas é relatada menos frequentemente. As manifestações clínicas são variadas e dependentes do local de acometimento. A incidência de angina mesentérica é rara. ${ }^{2}$

A terapêutica utilizada baseia-se sobretudo no uso de corticosteroides e imunossupressores, visto que alguns estudos observacionais relatam resposta de pacientes com AT ao uso de corticosteroides, metotrexato (MTX), azatioprina (AZA) e ciclofosfamida. ${ }^{1}$ As perspectivas medicamentosas em AT admitem maiores esperanças com a introdução da terapia biológica, apesar do pequeno número de pacientes já tratados.

A terapia medicamentosa freia a progressão da doença, mas as lesões vasculares já estabelecidas não respondem adequadamente, representando, portanto, um desafio propedêutico. Nesse contexto, a angioplastia transluminal percutânea com stents representa uma estratégia terapêutica de grande valia, apesar da reincidência de estenoses. ${ }^{3}$

\section{RELATO DE CASO}

Paciente 33 anos, sexo feminino, com mal-estar, febre, mialgia, cefaleia intensa, pulsátil, holocraniana e resistente a analgésicos, hipertensão arterial sistêmica de difícil controle, claudicação em membro inferior direito e dor abdominal de forte intensidade, a qual piorava após a alimentação. O nível pressórico aferido no membro superior direito foi $180 \times 60 \mathrm{mmHg}$, enquanto no membro superior esquerdo foi $100 \times 60 \mathrm{mmHg}$. Apresentava sopro sistólico no foco

Recebido em 28/1/2010. Aprovado após revisão em 24/1/2011. Os autores declaram a inexistência de conflitos de interesse.

Universidade Federal de Alagoas - UFAL.

1. Estudante de Medicina da UFAL

2. Médico-reumatologista da UFAL

Correspondência para: Luana Thayse Barros de Lima. Universidade Federal de Alagoas. Av. Brasil, 271, Poço, Maceió, Alagoas, Brasil. CEP: 57000-000. E-mail: luana_thayse@hotmail.com. 

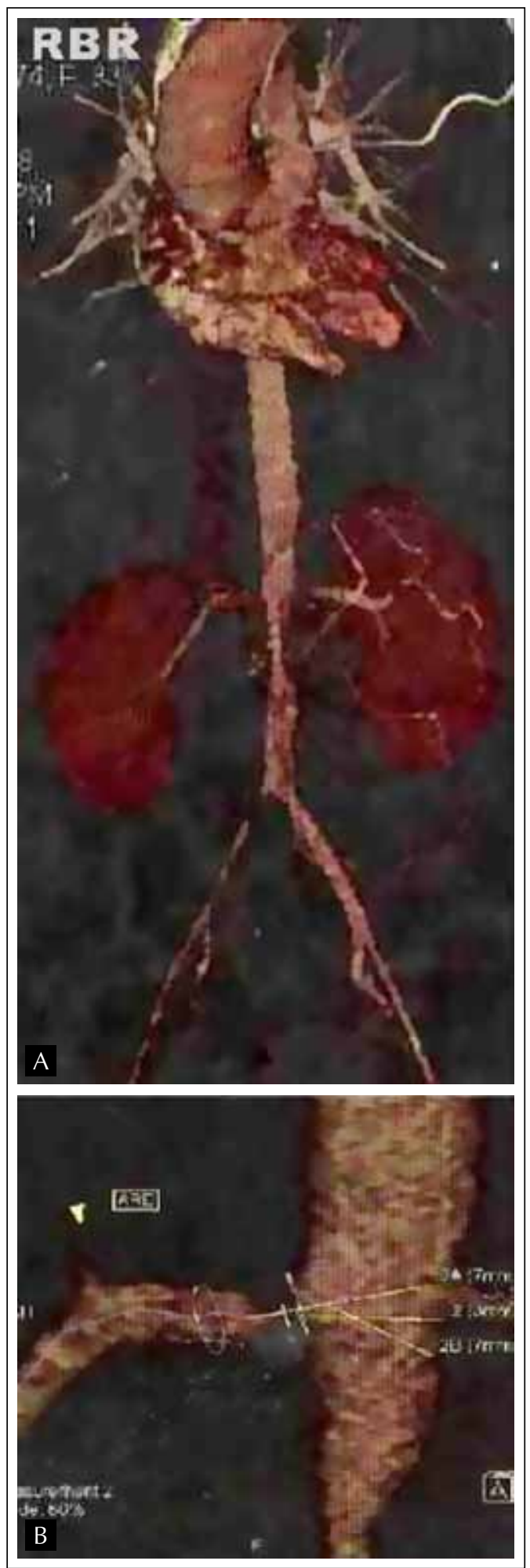

\section{Figura 1}

Tomografia computadorizada multislice. (A) Aneurisma da aorta ascendente, estenose da artéria ilíaca direita e estenose das artérias renais. (B) Estenose da artéria renal direita. aórtico e no território da artéria braquial esquerda, sem outras alterações ao exame físico; amilase, lípase, exame parasitológico de fezes, radiografia de tórax e ultrassonografia de abdome sem alterações significativas. $\mathrm{O}$ ecocardiograma exibiu dilatação aneurismática da aorta ascendente. A angiotomografia revelou aneurisma da aorta ascendente, estenose da artéria ilíaca comum direita, estenose das artérias renais e estenose da artéria mesentérica superior. Com o objetivo de controlar a doença foi introduzida a prednisona na dose de $1 \mathrm{mg} / \mathrm{kg} / \mathrm{dia}$.

Os achados da tomografia computadorizada multislice incluíram aneurisma de aorta ascendente, estenose de artéria ilíaca comum direita e estenose de artérias renais (Figuras 1A e 1B).

Frente aos achados angiotomográficos, associados às repercussões clínicas, a angioplastia transluminal percutânea foi realizada, com colocação de stents na artéria ilíaca comum direita, em ambas as artérias renais e na artéria mesentérica superior. A angiografia pré-angioplastia demonstrou estenose de tronco celíaco e artéria mesentérica superior (Figura 2A), além de estenose de artéria ilíaca comum direita. $\mathrm{O}$ controle final demonstrou a melhora da vascularização após a colocação dos stents (Figura 2B).

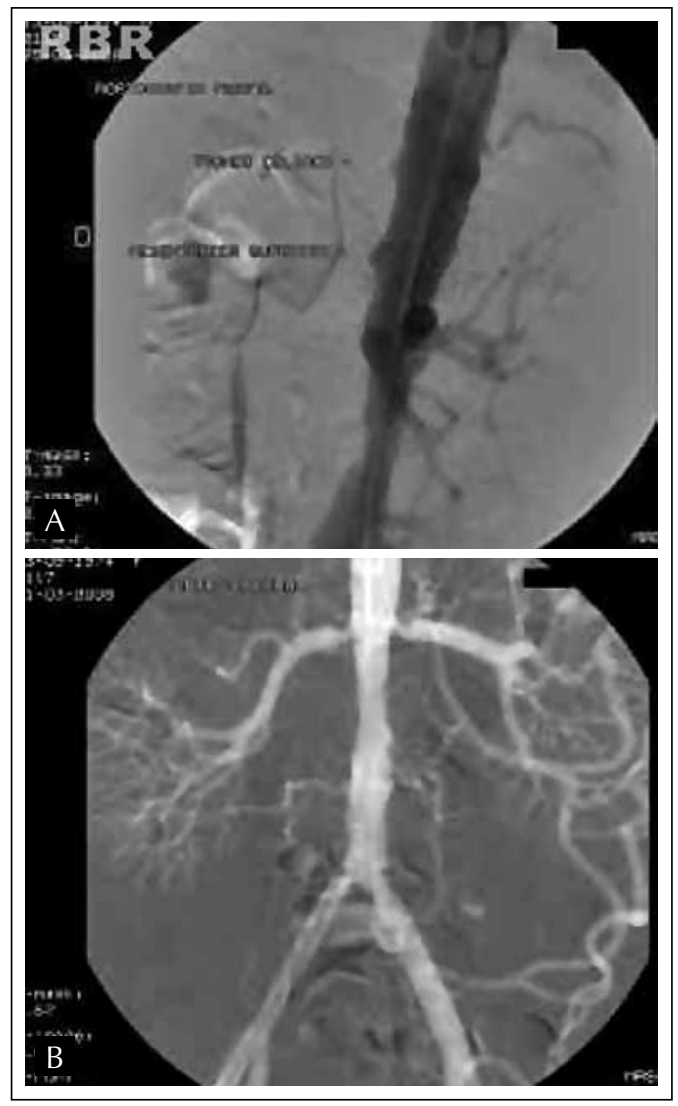

Figura 2

Angiografia. (A) Angiografia pré-angioplastia transluminal. (B) Controle final pós-angioplastia transluminal. 


\section{DISCUSSÃO}

O achado angiotomográfico de estenose de artéria mesentérica superior, associado ao quadro álgico abdominal persistente, embasou a conduta intervencionista através da angioplastia transluminal percutânea com a colocação de stent. Embora haja evidências na literatura de reestenose, ${ }^{3}$ a revascularização percutânea representa uma alternativa consistente e com melhor prognóstico quando a doença estiver clinicamente controlada. $\mathrm{O}$ acompanhamento clínico deve estender-se por período prolongado, principalmente em função do pequeno número de casos relatados. ${ }^{3}$

A aterosclerose é a causa mais comum de isquemia mesentérica; ${ }^{2}$ as trombofilias e vasculites primárias constituem causas menos frequentes. ${ }^{4} \mathrm{O}$ acometimento desse vaso em portadores de arterite de Takayasu representa, por si, um fator de mal prognóstico, uma vez que pode cursar de forma fulminante e refratária ao tratamento clínico ou cirúrgico. ${ }^{2}$ Constituem consequências temíveis da isquemia mesentérica o infarto mesentérico, a sepse e a morte. ${ }^{2,4}$

Embora seja pouco frequente, é importante pensar em angina mesentérica em todo paciente com arterite de Takayasu que evolua com dor abdominal, principalmente que piora após as refeições. ${ }^{4} \mathrm{~A}$ angiografia é um método de imagem direto que permite avaliar o comprometimento dos vasos. ${ }^{5}$ Entretanto a angiotomografia mostra ser uma alternativa diagnóstica não invasiva. $\mathrm{O}$ tratamento ideal é a corticoterapia em doses plenas e, perante estenose com acentuada redução do fluxo, deve-se proceder à colocação de stents, sob a precaução de realizar o tratamento cirúrgico preferencialmente no período de inatividade da doença, quando o emprego da técnica está associado a resultados mais favoráveis. ${ }^{6}$

Estudos recentes ressaltam a angioplastia percutânea como um método eficaz e duradouro no tratamento de estenoses desencadeadas por vasculites primárias ${ }^{7,8}$ - o índice de reestenoses varia de $20 \%$ a $44 \%{ }^{3,7}$ No entanto tal complicação pode ser abordada com uma nova intervenção endovascular sem complicações significativas. ${ }^{7}$ A técnica aberta de revascularização também é uma possibilidade terapêutica com bons resultados a longo prazo. ${ }^{8}$

\section{REFERENCES}

\section{REFERÊNCIAS}

1. Souza AWS, Neves RMS, Oliveira KR, Sato EI. Tratamento da arterite de Takayasu. Rev Bras Reumatol 2006; 46:2-7.

2. Simon S, Schittko G, Bösenberg H, Holl-Ulrich K, SchwarzEywill M. Fulminant course of a Takayasu's arteritis and rare mesenteric arterial manifestation. Zeitschrift für Rheumatologie 2006; 65(6):520-2.

3. Almeida GT, Barbosa BML, Biatto JFP, Casaroto E, Loures MAAR. Tratamento coadjuvante da arterite de Takayasu através de angioplastia transluminal percutânea com stents: relato de caso e revisão de literatura. Rev Bras Reumatol 2008; 48:118-21.

4. Cornejo R, Gatica H, Segovia E, Cortés C. Intestinal necrosis as clinical presentation of Takayasu arteritis. Revista Médica do Chile 2002; 130(10):1159-64.

5. Tumelero RT, Teixeira JCC, Duda NT, Tognon AP, Rossato M. Tratamento percutâneo multivaso da arterite de Takayasu. Arq Bras Cardiol 2006; 87(5):182-8.

6. Gelape CL, Alvarenga FC, Figueroa CCS, Ribeiro ALP. Tratamento de estenose de tronco de coronária esquerda na arterite de Takayasu. Rev Bras Reumatol 2007; 47:390-3.

7. Rits Y, Oderich GS, Bower TC, Miller DV, Cooper L, Ricotta JJ $2^{\text {nd }}$ et al. Interventions for mesenteric vasculitis. J Vasc Surg 2010; 51(2):392-400.

8. Numano F. Differences in clinical presentation and outcome in different countries for Takayasu's arteritis. Curr Opin Rheumatol 1997; 9:12-5.

9. Lee BB, Laredo J, Neville R, Villavicencio JL. Endovascular management of takayasu arteritis: is it a durable option? Vascular 2009; 17(3):138-46. 